\title{
Trial number and compound stimuli temporal relationship as joint determinants of second-order conditioning and conditioned inhibition
}

\author{
STEVEN STOUT \\ State University of New York, Binghamton, New York \\ MARTHA ESCOBAR \\ Auburn University, Auburn, Alabama \\ and \\ RALPH R. MILLER \\ State University of New York, Binghamton, New York
}

\begin{abstract}
Two conditioned lick suppression experiments with rats used feature-negative training (A-footshock trials intermixed with nonreinforced XA presentations) to analyze the role of the number of XA compound presentations and the temporal relationship of the elements within the compound (simultaneous or serial) as determinants of the resulting behavioral control. Second-order conditioning (i.e., excitatory behavioral control by $\mathrm{X}$ ) was observed to decline as the number of XA compound trials was increased. This decline was more rapid if X and A were presented simultaneously, as opposed to serially (i.e., $\mathrm{X}$ before A; Experiment 1). Conditioned inhibition to X, as assessed by a summation test (Experiment 1) and a retardation test (Experiment 2), increased with the number of XA trials and did so more quickly for simultaneous than for serial pairings of $\mathrm{X}$ and $\mathrm{A}$. The results help to clarify previously discrepant findings regarding factors that promote excitation versus inhibition with this protocol.
\end{abstract}

A basic discriminative conditioning protocol, first experimentally studied by Pavlov (1927), involves pairing an initially neutral stimulus (A) with an unconditioned stimulus (US) on some conditioning trials, while on others presenting that same stimulus without the US and in compound with a second neutral stimulus (X). Of interest in studies employing this $\mathrm{A}-\mathrm{US} / \mathrm{X}-\mathrm{A}$, or feature-negative, protocol is the type of responding, if any, elicited by stimulus X. Under some circumstances, feature-negative training results in subjects' responding to $\mathrm{X}$ as if it signals the impending occurrence of the US, despite X's never having been paired directly with the US. In this case, secondorder conditioning is said to have occurred. However, with apparently minor procedural variations, the featurenegative paradigm can result in the subject's responding to $\mathrm{X}$ as if it expects that the US will not be immediately forthcoming. In this case, conditioned inhibition is said to have

Support for this research was provided by NIMH Grant 33881, and support for preparation of the manuscript was provided by NIMH Grant 064420. We thank Jeffrey Amundson, Francisco Arcediano, Daniel Burger, Oskar Pineño, Kouji Urushihara, and Daniel Wheeler for their insightful advice concerning all aspects of this research. Special thanks are due Raymond Chang, Jim Esposito, and Jennifer Kelschenbach for assistance in running the animals in what has become quasi-affectionately known as the "Monster Study." Inquiries concerning this research should be addressed to R. R. Miller, Department of Psychology, SUNYBinghamton, Binghamton, NY 13902-6000 (e-mail: rmiller@binghamton. edu). occurred. Surprisingly, despite the obvious similarities between the procedures yielding these behaviorally opposite outcomes, there has been little systematic research to determine when second-order conditioning or conditioned inhibition (or both) will result from this protocol.

An investigation into the factors responsible for excitation or inhibition with this paradigm should ideally employ three dependent measures. To demonstrate that stimulus $\mathrm{X}$ is a second-order excitor requires that its responding be compared with that of another nonreinforced stimulus from a control condition providing little basis for excitation to develop (e.g., unpaired X and A, or unpaired $\mathrm{A}$ and the US; Holland \& Rescorla, 1975; Rizley \& Rescorla, 1972). In addition, most learning investigators accept that two separate tests, summation and retardation of acquisition, are necessary to establish whether $\mathrm{X}$ is a conditioned inhibitor (Rescorla, 1969). In the summation test, the conditioned response elicited by the simultaneous presentation of the putative inhibitor (X) and an independently trained excitatory conditioned stimulus (CS) is compared with the response elicited by the presentation of the independently trained excitatory CS alone. Reduced responding to the stimulus compound relative to the excitor alone provides evidence for conditioned inhibition, if care is taken to control for other response-decrementing processes, such as generalization decrement (Papini \& Bitterman, 1993). In the retardation procedure, the potentially inhibitory stimulus $(\mathrm{X})$ is reinforced, and excitatory re- 
sponding to $\mathrm{X}$ is compared with that of a reinforced stimulus that did not receive inhibitory training. Slowed acquisition of excitation to $\mathrm{X}$ relative to the control stimulus would suggest that, at the outset of reinforced training, $X$ had informed the subject that the US would not be forthcoming (i.e., was inhibitory). Probably because of the logistical difficulty inherent in examining the effect of manipulations of any variable or combination of variables on responding in these three separate tests (i.e., excitation, summation, and retardation), they have rarely been used together, either within a single experiment or across otherwise identical experiments within the same experimental series. To our knowledge, only Yin, Barnet, and Miller (1994) have used the three-test strategy in examining the effect of a candidate controlling variable, number of AX trials, on the response potential of X. We again employed this technique in the present experiments.

In a few studies, an attempt has been made to explore the issue of feature-negative behavioral control of both excitation and inhibition without all three tests. In these studies, excitation to $\mathrm{X}$ has usually been inferred from responding to $\mathrm{X}$ prior to A's being presented (i.e., training has often involved a delay between onset of $X$ and onset of A). To assess the inhibitory response potential of CS X, these studies have measured changes in conditioned responding to the AX compound relative to A alone (i.e., discrimination learning; Maisiak \& Frey, 1977; Rashotte, Marshall, \& O'Connell, 1981). Excitatory responding to the XA compound is commonly observed to increase, before decreasing with more $\mathrm{X}-\mathrm{A}$ pairings (e.g., Kehoe, Feyer, \& Moses, 1981). The logic behind this strategy is that because A is rendered excitatory by reinforcement, a decline in responding to the XA compound must be due to the buildup of inhibition as a factor counteracting the excitation (Spence, 1936). A difficulty with this classic interpretation is that apparent inhibition can result from a perceptual stimulus configuration of $\mathrm{A}$ and $\mathrm{X}$. If the response decrement to the compound is due to a configuration of $\mathrm{A}$ and $\mathrm{X}$ (or the trace of $\mathrm{X}$ in the serial featurenegative procedure), $\mathrm{X}$ would not be expected to transfer its response-decrementing potential to other conditioned excitors, as it would if it were a conditioned inhibitor. Thus, studies that fail to test whether $\mathrm{X}$ reduces the response potential of an independently trained conditioned excitor provide, at best, indirect evidence of conditioned inhibition. Another difficulty with studies of this sort is the preclusion of substantial overlap between $\mathrm{X}$ and $\mathrm{A}$.

Another reason for the small number of systematic explorations of the determining conditions of second-order conditioning versus conditioned inhibition is that most modern theories of conditioning simply ignore secondorder conditioning and do not predict its existence (e.g., Gallistel \& Gibbon, 2000; Mackintosh, 1975; R. R. Miller \& Matzel, 1988; Pearce, 1987; Pearce \& Hall, 1980; Rescorla \& Wagner, 1972). An exception to this pattern is the extension of the Rescorla and Wagner model by Sutton and Barto (1981), which allows indirect retrieval of the
US through a mediating CS representation, resulting in second-order conditioning. Conditioned inhibition results from subsequent pairings of CS X with the absence of an expected US. A similar strategy is employed in the more recent model of McLaren and Mackintosh (2000).

Despite the logistical difficulty and theoretical disinterest in the determinants that differentiate conditioned inhibition and second-order excitation, a few variables have been claimed to be important. One is whether the two types of trials are presented in separate experimental phases or interspersed within the same phase. Textbooks often distinguish between the procedure in which all of the A-US trials precede the $\mathrm{X}-\mathrm{A}$ trials and the feature-negative procedure, in which the reinforced and the nonreinforced trial types are pseudorandomly interspersed, sometimes calling the former procedure second-order conditioning and the latter Pavlovian conditioned inhibition (Lieberman, 2000, and Papini, 2002, are among the many undergraduate textbooks that assert this distinction). Despite these common labels, Yin et al. (1994) found that whether the trial types were presented phasically or interspersed made little difference in whether second-order conditioning or conditioned inhibition was observed. Maisiak and Frey (1977) actually observed behavior opposite to that suggested by the procedural labels. With their preparation and parameters, the interspersed procedure promoted secondorder conditioning, whereas the phasic procedure (all A-US trials before the $\mathrm{X}-\mathrm{A}$ trials) promoted conditioned inhibition. Despite the discrepancies with these two procedures, the third method of administering the reinforced and nonreinforced trials, sensory preconditioning $(\mathrm{X}-\mathrm{A}$ then A-US) has only been observed to produce either excitation or little responding.

Pavlov $(1927$, pp. 33, 69) contended that the chief variable determining whether the feature-negative protocol results in excitation or inhibition is the temporal relationship between $\mathrm{X}$ and $\mathrm{A}$. He maintained that simultaneous pairings of $\mathrm{X}$ and $\mathrm{A}$ facilitate conditioned inhibition, whereas serial pairings, with X preceding A, promote second-order conditioning. Subsequent investigations have clouded this picture by showing that either type of behavioral control (excitation or inhibition) can result from either temporal arrangement. For example, studies examining negative occasion setting (e.g., Rescorla, 1985) often yield test stimuli that pass summation and retardation tests and, thus, are operationally indicative of conditioned inhibition. ${ }^{1}$ In these studies, X usually precedes A. On the other hand, some investigators have successfully used simultaneous pairings to obtain second-order conditioning (e.g., Rescorla, 1982). Further challenging Pavlov's contention was Maisiak and Frey's (1977) study explicitly examining the effect of temporal relationship of X and A on secondorder conditioning and conditioned inhibition in a test of retarded acquisition of responding to $\mathrm{X}$ when it was reinforced. In this study, second-order conditioning was observed to result from trials in which $\mathrm{X}$ and A substantially overlapped (with X onset preceding A onset), but not from 
serial $\mathrm{X}-\mathrm{A}$ trials (with $\mathrm{X}$ termination and $\mathrm{A}$ onset simultaneous); however, weak conditioned inhibition resulted from the serial procedure.

Conflicting reports about the effect of the temporal arrangement of $\mathrm{X}$ and $\mathrm{A}$ imply that this variable interacts with another variable or variables to determine whether net excitation or inhibition will result. One candidate interacting variable is the number of $\mathrm{X}-\mathrm{A}$ pairings. Studies in which the influence of the number of $\mathrm{X}-\mathrm{A}$ trials has been examined have shown that, under similar circumstances, second-order excitation is more likely with few $\mathrm{X}-\mathrm{A}$ trials and conditioned inhibition is more likely after many X-A trials (e.g., Herendeen \& Anderson, 1968; Rizley \& Rescorla, 1972; Yin et al., 1994). Thus, inhibitory conditioning might be inevitable given enough $\mathrm{X}-\mathrm{A}$ trials - provided that so many $\mathrm{X}-\mathrm{A}$ trials are not presented that the association between A and the US is extinguished. Other parameters of training, such as the temporal arrangement of X and A (Maisiak \& Frey, 1977) or the proportion of X trials in which X and A are jointly presented (Rashotte et al., 1981), might also modulate the rate at which X becomes inhibitory and, possibly, the magnitude of excitation and inhibition achieved during this transition. If this were the case, then depending on the number of $\mathrm{X}-\mathrm{A}$ pairings preceding the test of the target CS, one could observe an advantage for either temporal arrangement in the amount of second-order conditioning or conditioned inhibition produced. Kehoe et al. (1981) examined the effect of temporal contiguity of $\mathrm{X}$ and $\mathrm{A}$ on the maximum amplitude of conditioned excitation achieved by $\mathrm{X}$ during featurenegative training. They used a procedure in which the duration of an empty trace period between $\mathrm{X}$ and $\mathrm{A}$ was varied. With this procedure, responding to $\mathrm{X}$ could be measured throughout acquisition, which is not possible if there is substantial or complete overlap between X and A. Kehoe et al. found shorter traces to facilitate the maximum level of second-order excitation achieved by $\mathrm{X}$ before secondorder conditioning eventually declined. Extrapolating their findings to the experiments reported here, we expected a similar result when the temporal contiguity of $X$ and A was manipulated. Simultaneous pairings might lead to more second-order conditioning early in training, followed by a transition to a greater level of conditioned inhibition than do serial pairings. Given that the rate of acquisition in excitatory conditioning is facilitated by good temporal contiguity (i.e., a zero gap procedure; Gibbon \& Balsam, 1981), we thought that we might also observe the rate of the transition in type and magnitude of behavior control (from excitatory to inhibitory) to depend on the temporal relationship between X and A. Just such a relationship was reported by Gibbs, Cool, Land, Kehoe, and Gormezano (1991), but they assessed only X-A intervals greater than zero (i.e., they did not examine simultaneous XA presentations), and they tested only for second-order conditioning.

The purpose of the present experiments, therefore, was to examine the possible interaction between the number of $\mathrm{X}-\mathrm{A}$ pairings and the temporal contiguity of $\mathrm{X}$ and $\mathrm{A}$ (serial or simultaneous) as determinants of X's response potential (excitatory or inhibitory). The present experiments differed from past examinations of the effects of temporal contiguity with the interspersed feature-negative preparation in at least two important respects: (1) by employing the three-test strategy and (2) by using X and A stimuli of equal duration in both conditions to avoid confounding stimulus durations with temporal contiguity, as past studies have done (e.g., Maisiak \& Frey, 1977; Rescorla, 1973). Both experiments used the conditioned lick suppression preparation with rat subjects, which received tests of second-order excitation (Experiment 1), summation (Experiment 1), or retardation of acquisition (Experiment 2) following identical manipulations of the independent variables. In both experiments, independent groups received 4,20 , or 100 pairings of $\mathrm{X}$ and $\mathrm{A}$, presented either serially (X before $\mathrm{A}$ ) or simultaneously. Two additional control groups received zero $\mathrm{X}-\mathrm{A}$ pairings during acquisition, and therefore, the temporal contiguity variable was not defined for this condition. All the animals received the same number of A-US pairings.

\section{EXPERIMENT 1}

The design for Experiment 1 is depicted in Table 1. Animals in 14 groups received pairings of a flashing light (A) and a footshock US. The first 12 groups listed in Table 1 also received pairings of a click train (X) and the light (A) throughout Phase $2 .^{2} \mathrm{~A}$ third of these 12 groups received 4 , a third 20, and a third $100 \mathrm{X}-\mathrm{A}$ pairings during Phase 2 . Two of the four groups given each number of $\mathrm{X}$-A pairings received $\mathrm{X}$ and $\mathrm{A}$ simultaneously, whereas the other two received $\mathrm{X}$ followed by A without a gap or overlap. Following this experience, a summation test for conditioned inhibition involved presenting $\mathrm{X}$ together with a transfer excitor (a tone; $\mathrm{T}$ ) to half the subjects or just the transfer excitor alone to the other half. A subsequent test for second-order excitation involved presenting $\mathrm{X}$ alone

Table 1 Experiment 1 Design Summary

\begin{tabular}{|c|c|c|c|c|c|}
\hline Group & Phase 1 & Phase 2 & Phase 3 & Test 1 & Test 2 \\
\hline Few/Sim/T & $\mathrm{T} \rightarrow \mathrm{US}$ & $\mathrm{A} \rightarrow \mathrm{US} / 4 \mathrm{XA}$ & $\mathrm{T} \rightarrow \mathrm{US}$ & $\mathrm{T}$ & $\mathrm{X}$ \\
\hline Few/Sim/XT & $\mathrm{T} \rightarrow \mathrm{US}$ & $\mathrm{A} \rightarrow \mathrm{US} / 4 \mathrm{XA}$ & $\mathrm{T} \rightarrow \mathrm{US}$ & XT & NA \\
\hline $\mathrm{Int} / \mathrm{Sim} / \mathrm{T}$ & $\mathrm{T} \rightarrow \mathrm{US}$ & $\mathrm{A} \rightarrow \mathrm{US} / 20 \mathrm{XA}$ & $\mathrm{T} \rightarrow \mathrm{US}$ & $\mathrm{T}$ & $\mathrm{X}$ \\
\hline Int/Sim/XT & $\mathrm{T} \rightarrow \mathrm{US}$ & $\mathrm{A} \rightarrow \mathrm{US} / 20 \mathrm{XA}$ & $\mathrm{T} \rightarrow \mathrm{US}$ & XT & NA \\
\hline Many/Sim/T & $\mathrm{T} \rightarrow \mathrm{US}$ & $\mathrm{A} \rightarrow \mathrm{US} / 100 \mathrm{XA}$ & $\mathrm{T} \rightarrow \mathrm{US}$ & $\mathrm{T}$ & $X$ \\
\hline Many/Sim/XT & $\mathrm{T} \rightarrow \mathrm{US}$ & $\mathrm{A} \rightarrow \mathrm{US} / 100 \mathrm{XA}$ & $\mathrm{T} \rightarrow \mathrm{US}$ & XT & NA \\
\hline Few/Ser/T & $\mathrm{T} \rightarrow \mathrm{US}$ & $\mathrm{A} \rightarrow \mathrm{US} / 4 \mathrm{X} \rightarrow \mathrm{A}$ & $\mathrm{T} \rightarrow \mathrm{US}$ & $\mathrm{T}$ & $X$ \\
\hline Few/Ser/XT & $\mathrm{T} \rightarrow \mathrm{US}$ & $\mathrm{A} \rightarrow \mathrm{US} / 4 \mathrm{X} \rightarrow \mathrm{A}$ & $\mathrm{T} \rightarrow \mathrm{US}$ & XT & NA \\
\hline Int/Ser/T & $\mathrm{T} \rightarrow \mathrm{US}$ & $\mathrm{A} \rightarrow \mathrm{US} / 20 \mathrm{X} \rightarrow \mathrm{A}$ & $\mathrm{T} \rightarrow \mathrm{US}$ & $\mathrm{T}$ & $\mathrm{X}$ \\
\hline Int/Ser/XT & $\mathrm{T} \rightarrow \mathrm{US}$ & $\mathrm{A} \rightarrow \mathrm{US} / 20 \mathrm{X} \rightarrow \mathrm{A}$ & $\mathrm{T} \rightarrow \mathrm{US}$ & XT & NA \\
\hline Many/Ser/T & $\mathrm{T} \rightarrow \mathrm{US}$ & $\mathrm{A} \rightarrow \mathrm{US} / 100 \mathrm{X} \rightarrow \mathrm{A}$ & $\mathrm{T} \rightarrow \mathrm{US}$ & $\mathrm{T}$ & $\mathrm{X}$ \\
\hline Many/Ser/XT & $\mathrm{T} \rightarrow \mathrm{US}$ & $\mathrm{A} \rightarrow \mathrm{US} / 100 \mathrm{X} \rightarrow \mathrm{A}$ & $\mathrm{T} \rightarrow \mathrm{US}$ & XT & NA \\
\hline None/T & $\mathrm{T} \rightarrow \mathrm{US}$ & $\mathrm{A} \rightarrow \mathrm{US}$ & $\mathrm{T} \rightarrow \mathrm{US}$ & $\mathrm{T}$ & $X$ \\
\hline None/XT & $\mathrm{T} \rightarrow \mathrm{US}$ & $\mathrm{A} \rightarrow \mathrm{US}$ & $\mathrm{T} \rightarrow \mathrm{US}$ & XT & NA \\
\hline
\end{tabular}

Note-A, light; X, clicks; T, tone; US, $1.0 \mathrm{~mA}, 0.5$-sec footshock; Few, 4 (0.5/day) X-A trials; Intermediate (Int), 20 (2.5/day) X-A trials; Many, 100 (12.5/day) X-A trials; None, 0 X-A trials; Ser, serial; Sim, simultaneous; $\rightarrow$, followed by; NA, not applicable. 
to those subjects that had been tested with T-alone in the summation test on the prior day of testing. An assumption underlying this strategy was that, although generalized extinction from testing with CS T to CS X may have occurred, it should have generalized uniformly given uniform test exposure to CS T (i.e., it did not transfer differentially to $\mathrm{X}$ as a function of X's prior treatment). Two additional groups of rats received only pairings of A and the US during Phase 2; that is, they received no $\mathrm{X}-\mathrm{A}$ pairings. These groups were included in order to control for several processes that might affect responding to stimulus $\mathrm{X}$ independent of the discrimination training. These include pseudoconditioning and orienting responses, which might mimic any positive summation observed, and generalization decrement and external inhibition, which might mimic any negative summation observed. Because of the large number of groups involved, the classic controls for secondorder conditioning (unpaired A and the US; unpaired X and A) were not included in this experiment. However, our laboratory has shown that excitatory responding elicited by the second-order CS obtained with this procedure to be true second-order conditioning, when compared with these control conditions (Yin et al., 1994).

\section{Method}

\section{Subjects}

The subjects were 84 male and 84 female, experimentally naive, Sprague-Dawley derived rats, bred in our colony. Males weighed from 187 to $363 \mathrm{~g}$. Females weighed from 160 to $262 \mathrm{~g}$. They were individually housed in wire-mesh cages in a vivarium maintained on a 16:8-h light:dark cycle. All the subjects were handled for $30 \mathrm{sec}$ three times a week from weaning until the initiation of the study. Training occurred during the middle half of the light portion of the cycle. A progressive water deprivation schedule was imposed over the week prior to the beginning of the experiment until water availability was limited to $30 \mathrm{~min}$ per day. Each subject was randomly assigned to 1 of 14 groups $(n=12$; see the design summary in Table 1 for group names), counterbalanced within group for sex.

\section{Apparatus}

Six identical copies of each of two different types of experimental chambers were used in each of two identical training rooms, for a total of 12 chambers of each type. Chamber Rectangular (R) was a clear, Plexiglas, rectilinear chamber, measuring $23.0 \times 8.5 \times 12.5 \mathrm{~cm}$ $(1 \times \mathrm{w} \times \mathrm{h})$. The floor was constructed of 0.48 -cm-diameter stainless steel rods, spaced $1.5 \mathrm{~cm}$ apart, center to center. The rods were connected by NE- 2 neon bulbs that allowed a constant-current footshock to be delivered by means of a high-voltage AC circuit in series with a $1.0-\mathrm{M} \Omega$ resistor. Each copy of Chamber $\mathrm{R}$ was housed in a separate environmental isolation chest, which was dimly illuminated by a $2-\mathrm{W}$ (nominal at $120 \mathrm{VAC}$ ) incandescent bulb driven at $60 \mathrm{VAC}$. The houselight was mounted on the ceiling of the environmental chest, approximately $26 \mathrm{~cm}$ from the center of the experimental chamber.

Chamber V-shaped (V) was a 22.1-cm-long box in the shape of a vertical truncated $-\mathrm{V}$ shape $(28 \mathrm{~cm}$ height, $21 \mathrm{~cm}$ wide at the top, $5.25 \mathrm{~cm}$ wide at the bottom). The floor and sides were constructed of stainless steel sheets, and the ceiling was constructed of clear Plexiglas. The floor of each chamber consisted of two parallel metal plates, each $2.0 \mathrm{~cm}$ wide, with a $1.1-\mathrm{cm}$ gap between them. Each Vshaped chamber was housed in its own environmental isolation chest, which was dimly illuminated by a $7.5-\mathrm{W}$ (nominal at 120 VAC) incandescent houselight driven at $60 \mathrm{VAC}$ mounted on an inside wall of the environmental chest, approximately $30 \mathrm{~cm}$ from the center of the experimental chamber. The light entering the animal chamber was primarily that reflected from the roof of the environmental chest, which was constructed of white insulating material. The light intensities inside the animal enclosures in the two types of chambers were approximately equal, due to the differences in opaqueness of the walls in Chambers R and V.

Each chamber ( $\mathrm{R}$ and $\mathrm{V}$ ) could be equipped with a water-filled lick tube that extended $1 \mathrm{~cm}$ from the rear of a cylindrical niche, $4.5 \mathrm{~cm}$ in diameter, which was left-right centered in one short wall, with its axis perpendicular to the wall, and was positioned with its center $4.25 \mathrm{~cm}$ above the floor of the chamber. A horizontal infrared photobeam traversed each niche parallel to the wall on which the niche was mounted, $1 \mathrm{~cm}$ in front of the lick tube. In order to drink from the tube, the subjects had to insert their heads into the niche, thereby breaking the infrared photobeam. Thus, the time during which the subjects had their heads in the niche with the water tube could be monitored. Ordinarily, they did this only when they were drinking. Disruption of ongoing drinking by a test stimulus served as our dependent variable.

Each chamber ( $\mathrm{R}$ and $\mathrm{V}$ ) was also equipped with two speakers widely separated on the inside walls of the environmental chest. Each speaker could deliver a different auditory stimulus, which here consisted of a complex tone composed of two high frequencies (3000 and $3200 \mathrm{~Hz}$, presented simultaneously) and a click train $(6 / \mathrm{sec})$. Ventilation fans in each chest provided a constant $74-\mathrm{dB}$ (Cscale) background noise. A flashing light stimulus was provided by a $25-\mathrm{W}$ bulb (Chamber R) or by a $100-\mathrm{W}$ bulb (Chamber V), both nominal at $120 \mathrm{VAC}$ but driven at $60 \mathrm{VAC}$. The bulbs were mounted on an inside wall of the environmental chest, approximately $30 \mathrm{~cm}$ from the center of the experimental chamber. Due to differences in the opaqueness of the walls of Chambers $\mathrm{R}$ and V, these two visual stimuli were of similar intensity inside the animal chambers. When presented, the light flashed $(0.25 \mathrm{sec}$ on $/ 0.25 \mathrm{sec}$ off $)$. The tone $(8 \mathrm{~dB}$ above background) served as the transfer stimulus (T), the clicks ( $8 \mathrm{~dB}$ above background) served as the second-order stimulus $(\mathrm{X})$, and the flashing light served as the first-order stimulus (A). The US consisted of a $0.5-\mathrm{sec}, 1.0-\mathrm{mA}$ footshock.

\section{Procedure (See Table 1)}

Acclimation. On Day 1, all the subjects were exposed to the eventual test context (Context 1) for 60 min, during which time the lick tubes were present. For half the subjects in each group, Context 1 was Chamber R, and for the other half, it was Chamber V. The purpose of this acclimation session was to allow the subjects to locate and adapt to the lick tubes. No nominal stimuli were presented.

Preexposure. On Day 2, the subjects were exposed to Context 2 for $60 \mathrm{~min}$, during which time the lick tubes were removed. Context 2 was always different from the physical chamber, $\mathrm{R}$ or $\mathrm{V}$, that served as Context 1 . The lick tubes remained absent throughout all subsequent Context 2 experience in order to avoid spurious pairings of CSs or USs with licking. CS X (clicks) was presented to all the subjects at 7,18,37, and $48 \mathrm{~min}$ into the session. CS X and all other CSs in the experiment were of 10 -sec duration. The purpose of preexposing the animals to the clicks was to reduce possible external inhibition by the click during testing in the none conditions.

Phase 1: transfer excitor training. On Day 3 , two $\mathrm{T} \rightarrow$ US pairings were given to all the subjects in a 1-h session in Context 2 . The footshock US began $0.5 \mathrm{sec}$ before the termination of the 10 -sec tone (T) and ended with the termination of the tone. These pairings occurred at 15 and $45 \mathrm{~min}$ into the session.

Phase 2: feature-negative (A-US/XA) training. On Days 4-11, discrimination training occurred in Context 2. Session lengths were 60 -min, and all the groups received $6 \mathrm{~A} \rightarrow \mathrm{US}$ pairings per day. The subjects received different numbers of $\mathrm{X}-\mathrm{A}$ trials per session, depending on their group assignment. Those in the many condition received an average of $12.5 \mathrm{X}$-A pairings per session; those in the 
intermediate (int) condition received 2.5 per day, those in the few condition received 0.5 per session, and those in the none condition received zero per session. Thus, over the 8 days of Phase 2 , all the subjects received $48 \mathrm{~A} \rightarrow \mathrm{US}$ trials, and 100,20 , 4, or $0 \mathrm{X}-\mathrm{A}$ trials. Each group was exposed to one of two quasirandom training schedules on alternating days. The mean intertrial interval was 278, 1,426, and 7,190 sec for the many, int, and few conditions, respectively (range $= \pm 50 \%$ ). The US was always presented during the last $0.5 \mathrm{sec}$ of CS A. For the subjects in the simultaneous condition, $\mathrm{X}$ and A overlapped perfectly; for the subjects in the serial condition, onset of A coincided with termination of X (i.e., X preceded A with no gap or overlap).

Phase 3: transfer excitor training. On Day 12, the subjects received an additional day of T $\rightarrow$ US pairings in Context 2 according to the same schedule as that described in Phase 1.

Reacclimation. On Days 13 and 14, the subjects were placed into Context 1 with lick tubes present for a 60 -min session, during which no nominal stimuli were presented. These sessions were intended to reestablish a stable rate of baseline licking that might have been disrupted by the US presentations of Phase 1 .

Summation test. On Day 15, in order to assess conditioned inhibition, the subjects were placed into Context 1 with free access to the lick tube. Context 1 was used as the test context because of its relative associative neutrality. Time from placement of each subject in the apparatus to completion of 5 cumulative seconds of drinking was recorded. After the subjects drank for 5 cumulative seconds, the test stimulus was presented. Thus, all the subjects were licking at CS onset. The test stimulus was the tone (T) alone for half of the subjects and was a simultaneous compound of the tone and the click (XT) for the other half of the subjects. Dependent measures included the time to complete the first 5 cumulative seconds of drinking (preCS time) and the time from the onset of the test stimulus until completion of the second 5 cumulative seconds of drinking (CS time). The stimuli remained on for $12 \mathrm{~min}$ regardless of the time it took the subject to complete 5 cumulative seconds of drinking in the presence of the CS. This was done to equate the experience with CS T of all the subjects that would be tested with X on Day 16, so that differences in behavior on Day 16 could not be attributed to differential treatment on Day 15. All the subjects that took more than $60 \mathrm{sec}$ to complete their first 5 cumulative seconds of drinking on the test days (pre-CS times) were scheduled to be eliminated from the experiment for exhibiting unusually great generalization of fear to the test context. In practice, no subjects in this experiment met the criterion for elimination.

Excitation test. In order to assess second-order conditioning, the subjects that had been tested in Context 1 with the transfer stimulus alone on Day 15 were tested with the click alone on Day 16 (see Table 1). The test procedure was the same as the one used on Day 15.

\section{Results}

\section{Summation Test}

Group mean CS times from the summation test are depicted in Figure 1. Responding (i.e., conditioned lick suppression) of the subjects tested with the XT stimulus compound decreased as the number of previous $\mathrm{X}-\mathrm{A}$ pairings increased and decreased more quickly for the subjects in the simultaneous condition than for the subjects in the serial condition. Relative to the control groups, which were tested with the transfer stimulus (T) alone, negative summation was observed with 20 and 100 pairings for the simultaneous condition and with only 100 pairings for the serial condition. These impressions are supported by the following statistical comparisons.

Due to faulty equipment, test data for the summation test from 12 subjects were lost. These subjects included 1 subject in each group except Groups Few/Ser/T and Few/Ser/XT. One subject from Group Few/Sim/XT died during the experiment. Thus, final group sizes ranged from 10 to 12 . In order to approximate the assumptions of parametric statistics, all the scores in this and all the subsequent analyses were transformed to $\log$ (base 10) times to improve normality of the within-group distributions. We first analyzed pre-CS scores from the summation test with a one-way analysis of variance (ANOVA), to determine whether baseline performance was comparable across the 14 groups. The effect of group was nonsignificant ( $p>$ $.20)$. This variable was ignored in subsequent analyses.

CS times from the first 12 groups were analyzed (see Table 1) with a $3 \times 2 \times 2 \times 2$ factorial ANOVA to obtain

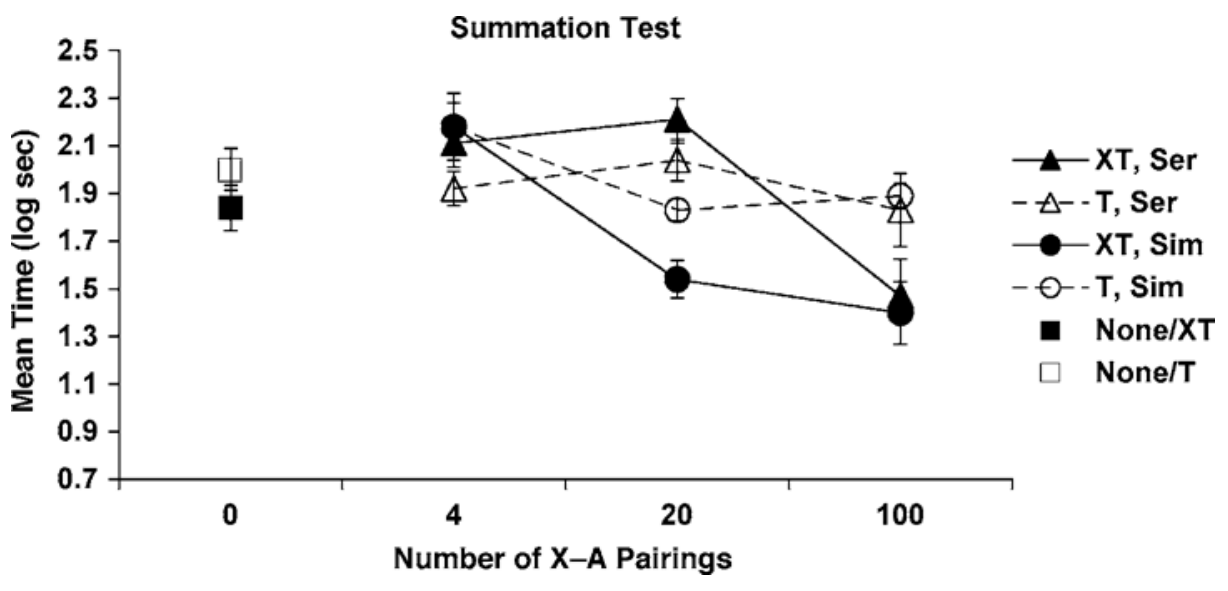

Figure 1. Mean times to resume drinking upon presentation of the test stimulus in the summation test of Experiment 1. Tests followed feature-negative training in which $X$ and $A$ were presented serially or simultaneously for $0,4,20$, or 100 pairings. Stimulus $T$ was the transfer excitor. Error bars represent $S E M$ s. 
the error term for the planned comparisons of each group tested with the compound (XT) and its matching control group tested with the transfer excitor alone $(\mathrm{T})$. The factors were number of trials ( 4 vs. 20 vs. 100), contiguity (serial vs. simultaneous), test stimulus (XT vs. T), and experimental room. The following effects were significant: number of trials $[F(2,109)=23.04, p<.001]$, experimental room $[F(1,109)=4.81, p<.001]$, the interaction of test stimulus and number of trials $[F(2,109)=6.41$, $p<.01]$, the interaction of test stimulus and contiguity $[F(1,109)=4.86, p<.05]$, and the interaction of number of trials and contiguity $[F(2,121)=12.34, p<.001]$. No other effects were significant (all $p \mathrm{~s}>.20$ ). Although the three-way interaction between number of trials, contiguity, and test stimulus did not achieve statistical significance, we felt that the three significant two-way interactions justified using planned comparisons to identify the source of these interactions.

The main planned comparisons were between T and XT at each level of contiguity and trial number $\left(M S_{\mathrm{e}}=0.114\right.$ for all comparisons). In the serial conditions, summation was significantly positive with few trials $[F(1,109)=$ $4.29, p<.05]$, nonsignificant with intermediate trials $(p>.25)$, and significantly negative with many trials $[F(1,109)=3.98, p<.05]$. Moreover, the simple interaction of trial number and test was significant in the serial condition $[F(2,56)=3.87, p<.05]$.

In the simultaneous conditions, summation was not significant with few trials $[F(1,109)=0.31, p>.57]$ but was significantly negative with intermediate $[F(1,109)=4.55$, $p<.05]$ and many $[F(1,109)=6.67, p<.01]$ trials. This pattern is consistent with conditioned inhibitions requiring fewer pairings to develop in the simultaneous than in the serial condition. Moreover, the simple interaction of trial number and test was significant in the simultaneous condition $[F(1,53)=3.40, p<.05]$. A comparison of responding in the two none groups tested for the possibility of external inhibition or generalization decrement's contributing to the negative summation that we observed. The mean differences, although consistent with decrement, were nonsignificant $(p>.21)$.

In the planned comparisons, we asked whether positive or negative summation was observed at each level of trial number $(4,20$, or 100) and temporal contiguity (serial or simultaneous). To allow us to draw more forceful conclusions about the relative size of the summation effects, we performed tests of the simple interaction of test stimulus and contiguity at each level of trial number. This test was equivalent to asking whether, at each level of trial number, the difference between T and XT (summation) varied as a function of contiguity. To enhance the power of the analyses, room was included as a factor, because it had been shown to exert a small but significant main effect on responding. Consistent with the graphical interpretation, and as suggested by the individual planned comparisons of $\mathrm{T}$ and $\mathrm{XT}$, contiguity and test stimulus interacted significantly only in the int condition $[F(1,36)=9.14, p<$ $.01]$, but not in the few or many conditions ( $p \mathrm{~s}>.34)$.
Taken together, the statistical analyses suggest that summation was similar in the 4- and 100-trial conditions but differed significantly in the 20-trial (int) condition. Notably, all tests involved simultaneous onset of X and T. Serial testing might have produced different outcomes, but our interest here was with conditioned inhibition, which is conventionally assessed with summation tests in which the elements onset simultaneously.

\section{Excitation Test}

The results from the second-order excitation test are presented in Figure 2. Excitatory responding declined with the number of $\mathrm{X}-\mathrm{A}$ pairings and did so most rapidly for the subjects in the simultaneous condition. Statistical analyses support these conclusions.

In a one-way ANOVA, we asked whether pre-CS scores differed reliably as a function of group membership. All the subjects previously tested with the transfer excitor alone contributed data for this test, including the $12 \mathrm{sub}-$ jects affected by the recording error on the previous day $(n=84)$. The effect of group was not significant $[F(6,77)=$ $1.93, p>.09]$, suggesting comparable baseline performances across groups.

A $7 \times 2$ (group $\times$ experimental room) ANOVA was conducted on CS scores to derive the error terms for the planned comparisons (because the goal of this analysis was to compare Group None/T with each remaining group, the error term could not be derived from the $2 \times 3$ factorial, as it had been in the analysis of the summation test). The effect of group was significant $[F(6,70)=12.74$, $\left.M S_{\mathrm{e}}=0.084, p<.001\right]$, and no other effects achieved significance $(p \mathrm{~s}>.36)$. The planned comparisons $\left(M S_{\mathrm{e}}=\right.$ 0.084 ) were between Group None/T and each of the remaining six groups. These tests determined whether responding to a stimulus subjected to the feature-negative treatment was sufficiently greater than that elicited by a stimulus that had not been subjected to that treatment. Responding of the subjects that received serial pairings of $\mathrm{X}$ and $\mathrm{A}$ was significantly excitatory following 4 trials $[F(1,70)=35.91, p<.001]$ and 20 trials $[F(1,70)=19.78$, $p<.001]$, but not following 100 trials $[F(1,70)=1.79$, $p>$.18]. In contrast, responding of subjects that received simultaneous pairings of $\mathrm{X}$ and $\mathrm{A}$ was excitatory following 4 trials $[F(1,70)=11.53, p<.001]$, but not 20 or 100 trials $(p s>.16)$. Consistent with the different levels of behavioral control observed at the intermediate level, Groups Int/Ser/T and Int/Sim/T differed significantly $[F(1,70)=9.31, p<.01]$.

\section{Discussion}

In summary, Experiment 1 demonstrated that secondorder excitatory conditioning decreased with the number of $\mathrm{X}-\mathrm{A}$ pairings (beyond the first few) and did so more rapidly when $\mathrm{X}$ and $\mathrm{A}$ were simultaneously, rather than serially, paired. Similarly, negative summation, indicative of conditioned inhibition, increased as a function of the number of $\mathrm{X}-\mathrm{A}$ pairings, and it did so within fewer pairings when $\mathrm{X}$ and $\mathrm{A}$ were simultaneously paired than when they 


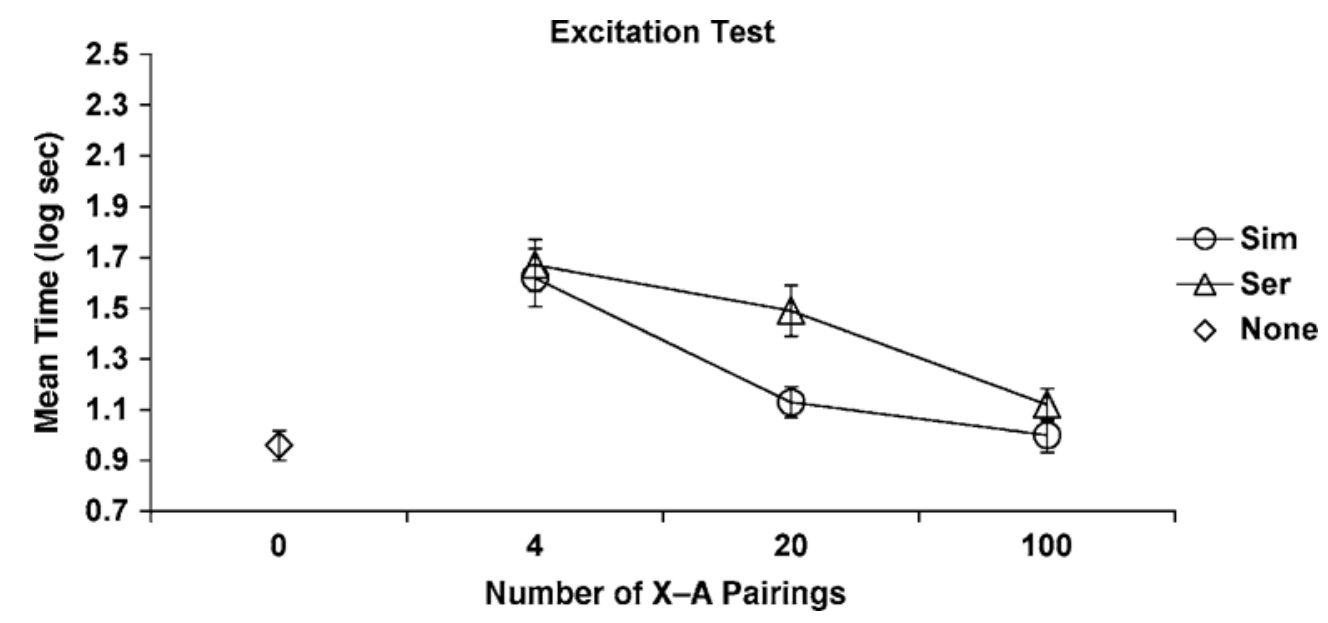

Figure 2. Mean times to resume drinking upon presentation of the test stimulus in the second-order excitation test of Experiment 1. The subjects tested were those tested with stimulus $T$ alone in the prior summation test. Error bars represent SEMs.

were serially paired. Excitatory and inhibitory responding were approximately equal following few and many pairings in both contiguity conditions.

The results of the negative summation test might illuminate previous findings that a serially trained negative feature (as X is sometimes called in this protocol) poorly transfers (if at all) its inhibitory potential to an independently trained transfer excitor. Holland and Lamarre (1984) and Lamarre and Holland (1987), for instance, found no such transfer, whereas previous studies from our laboratory have found some, although weak, negative transfer (e.g., Barnet \& Miller, 1996). Experiment 1 suggests that successful negative transfer develops as a function of the number of nonreinforced trials and, perhaps similarly, the amount of discrimination training to which the target stimulus has been subjected prior to the transfer test. As compared with a simultaneous negative feature, a serial feature apparently produces less negative summation following an intermediate number of pairings and statistically equivalent summation following many pairings. This suggests that inhibition might develop at different rates following each of these procedures.

\section{EXPERIMENT 2}

Experiment 2 complemented Experiment 1 by providing a retardation of excitatory stimulus control to assess conditioned inhibition. The first three phases of Experiment 2 were identical to those in Experiment 1. In Phase 4, the rats were given four reinforced pairings of CS X and the footshock US. Resistance to reinforcement was centrally measured by comparison of responding in the none condition with that in each of the remaining six groups.

\section{Method}

Subjects and Apparatus

The subjects were 42 male and 42 female experimentally naive Sprague-Dawley rats, bred in our colony. Males weighed from 199 to $354 \mathrm{~g}$. Females weighed from 170 to $252 \mathrm{~g}$. Housing, deprivation, and training conditions were identical to those in Experiment 1, except that in this experiment training took place in only one room. Each subject was randomly assigned to one of seven groups $(n=$ 12 ); see the design summary in Table 2 for group names, counterbalanced within group for sex.

\section{Procedure (See Table 2)}

Conditions of treatment during the acclimation and training Phases 1-3 were identical to the conditions described in Experiment 1 . Phase 4 treatment occurred on Day 13 and consisted of four pairings of the clicks (X) with the footshock US. The clicks were presented at 8,24, 37, and 56 min into the session. The 10-sec click presentations coterminated with the 0.5 -sec footshock presentations. Reacclimation took place on Days 14 and 15 and was identical to that described in Experiment 1. Testing of CS X took place on Day 16, following the same test procedure as that used in the secondorder excitation test of Experiment 1.

\section{Results and Discussion: Retardation Test}

The results of Experiment 2 are presented in Figure 3. Lick suppression times were high in Group None, in which no inhibition was expected because the subjects received no pairings of $\mathrm{X}$ and $\mathrm{A}$. This baseline for acquisition contrasts greatly with the low level of responding displayed by Group None/T in Experiment 1, which received the same treatment but lacked the $4 \mathrm{X}-\mathrm{US}$ pairings. Responding to $\mathrm{X}$ in the simultaneous and serial conditions following $4 \mathrm{X}$-US trials was approximately equal to the none condition, implying little resistance to acquisition (i.e., little retardation), and responding was low and approximately equal for both the simultaneous and the serial conditions following 100 trials, implying greater retardation of acquisition. The subjects in the serial condition exhibited little retardation after $20 \mathrm{X}-\mathrm{A}$ trials, in contrast to the apparently great retardation displayed by the subjects that received $20 \mathrm{X}-\mathrm{A}$ trials in the simultaneous condition.

No subjects were rejected due to high pre-CS times. Two subjects were eliminated during the course of the experiment due to illness, 1 from Group Few/Sim and 1 from 
Table 2

Experiment 2 Design Summary

\begin{tabular}{|c|c|c|c|c|c|}
\hline Group & Phase 1 & Phase 2 & Phase 3 & Phase 4 & Tes \\
\hline Few/Sim & $\mathrm{T} \rightarrow \mathrm{US}$ & $\mathrm{A} \rightarrow \mathrm{US} / 4 \mathrm{XA}$ & $\mathrm{T} \rightarrow \mathrm{US}$ & $\mathrm{X} \rightarrow \mathrm{US}$ & \\
\hline Few/Ser & $\mathrm{T} \rightarrow \mathrm{US}$ & $\mathrm{A} \rightarrow \mathrm{US} / 4 \mathrm{X} \rightarrow \mathrm{A}$ & $\mathrm{T} \rightarrow \mathrm{US}$ & $\mathrm{X} \rightarrow \mathrm{US}$ & \\
\hline Int/Sim & $\mathrm{T} \rightarrow \mathrm{US}$ & $\mathrm{A} \rightarrow \mathrm{US} / 20 \mathrm{XA}$ & $\mathrm{T} \rightarrow \mathrm{US}$ & $\mathrm{X} \rightarrow \mathrm{US}$ & \\
\hline Int/Ser & $\mathrm{T} \rightarrow \mathrm{US}$ & $\mathrm{A} \rightarrow \mathrm{US} / 20 \mathrm{X} \rightarrow \mathrm{A}$ & $\mathrm{T} \rightarrow \mathrm{US}$ & $\mathrm{X} \rightarrow \mathrm{US}$ & \\
\hline Many/Sim & $\mathrm{T} \rightarrow \mathrm{US}$ & $\mathrm{A} \rightarrow \mathrm{US} / 100 \mathrm{XA}$ & $\mathrm{T} \rightarrow \mathrm{US}$ & $\mathrm{X} \rightarrow \mathrm{US}$ & \\
\hline Many/Ser & $\mathrm{T} \rightarrow \mathrm{US}$ & $\mathrm{A} \rightarrow \mathrm{US} / 100 \mathrm{X} \rightarrow \mathrm{A}$ & $\mathrm{T} \rightarrow \mathrm{US}$ & $X \rightarrow U S$ & \\
\hline None & $\mathrm{T} \rightarrow \mathrm{US}$ & $\mathrm{A} \rightarrow \mathrm{US}$ & $\mathrm{T} \rightarrow \mathrm{US}$ & $\mathrm{X} \rightarrow \mathrm{US}$ & $\mathrm{X}$ \\
\hline
\end{tabular}

Note-A, light; X, clicks; T, tone; US, $1.0 \mathrm{~mA}, 0.5$-sec footshock; Few, $4(0.5 /$ day) X-A trials; Intermediate (Int), 20 (2.5/day) X-A trials; Many, 100 (12.5/day) X-A trials; None, 0 X-A trials; Ser, serial; Sim, simultaneous; $\rightarrow$, followed by.

Group Int/Sim. Analysis of pre-CS times indicated no effect of group $(p>.21)$, suggesting comparable baseline performance across groups.

A one-way ANOVA indicated that CS times were significantly affected by group $\left[F(6,74)=17.45, M S_{\mathrm{e}}=\right.$ $0.18, p<.001]$. The planned comparisons were between Group None and each of the remaining groups. They addressed the degree to which each group exhibited more or less retardation than the control group (None). Consistent with the appearance of Figure 3, Groups Few/Ser and Int/Ser did not differ from the control group $(p \mathrm{~s}>.32)$, but group Many/Ser did $[F(1,74)=17.45, p<.001]$. Group Few/Sim did not differ from the control $(p>.75)$, but Group Int/Sim did $[F(1,74)=22.25, p<.001]$, as did Group Many $/ \operatorname{Sim}[F(1,74)=21.70, p<.001]$. Moreover, in an ANOVA restricted to the experimental groups (all groups except Group None), the interaction of contiguity and trial number was significant $[F(2,63)=13.40, p<$ $.001]$. Groups Int $/$ Ser and Int $/$ Sim differed $[F(1,63)=$ $32.22, p<.001]$. Taken together, these analyses are consistent with retardation of conditioned responding decreasing with the number of $\mathrm{X}-\mathrm{A}$ trials in both contiguity conditions, but doing so more quickly in the simultaneous than in the serial condition.

\section{GENERAL DISCUSSION}

The present experiments demonstrated that, in a featurenegative discrimination (i.e., A-US/X-A), the temporal arrangement of the second-order $\mathrm{CS}(\mathrm{X})$ with respect to the first-order CS (A) interacted with the number of times that they were paired to determine whether excitation or inhibition was observed. Similar levels of conditioned excitation were observed following 4 simultaneous or serial pairings of X and A. Following 100 simultaneous or serial pairings of $\mathrm{X}$ and $\mathrm{A}$, both temporal relationships produced similar levels of conditioned inhibition, as measured by both retardation and summation tests. However, with 20 pairings a serial arrangement led to second-order excitation (Experiment 1), whereas a simultaneous arrangement led to conditioned inhibition, as assessed by both summation (Experiment 1) and retardation (Experiment 2) tests. Presumably, because of their superior contiguity, simultaneous $\mathrm{X}-\mathrm{A}$ pairings are more effective in promoting an $\mathrm{X}-\mathrm{A}$ association than are serial $\mathrm{X}-\mathrm{A}$ pairings; hence, it takes fewer simultaneous pairings to achieve the same behavioral result (i.e., the progression from second-order conditioning to conditioned inhibition). These results are at least partially consistent with Pavlov's (1927, pp. 33, 69) suggestion that a serial procedure favors the development of second-order conditioning; under the present conditions, second-order conditioning lasted for more pairings of X and A. However, inconsistent with Pavlov's suggestion, conditioned inhibition seems to be inevitable, given a sufficient number of $\mathrm{X}-\mathrm{A}$ pairings, with either procedure. The results were not consistent with our hypothesis that the simultaneous condition might produce greater excitation early and greater inhibition late in training,

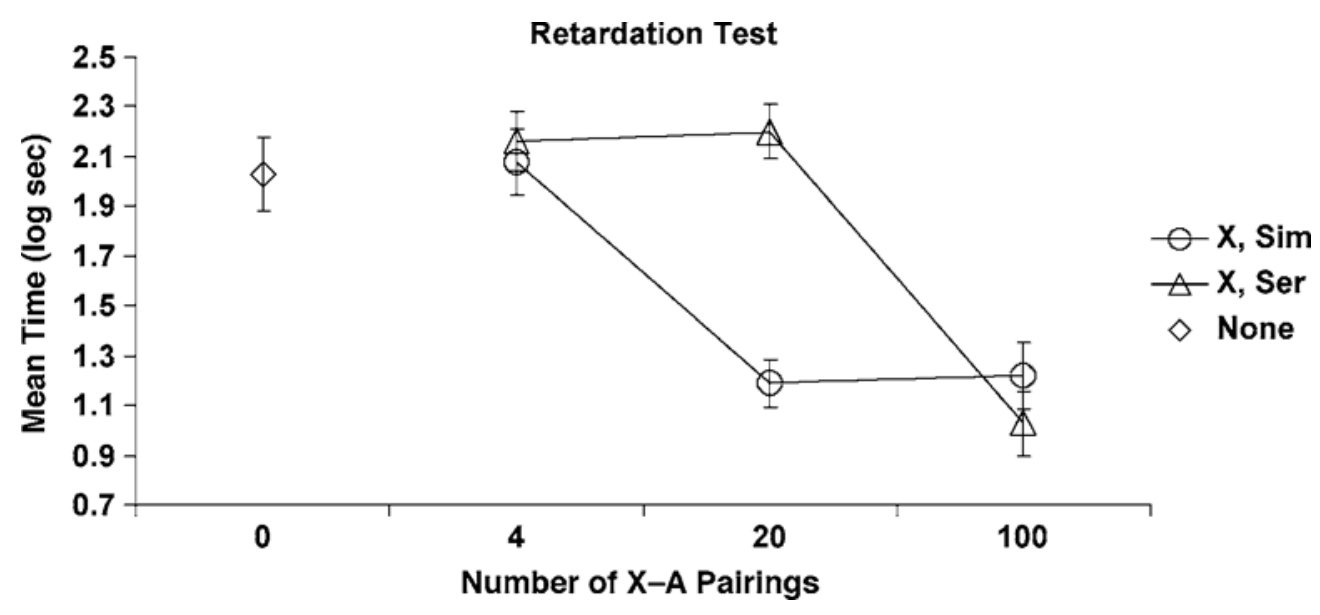

Figure 3. Mean times to resume drinking upon presentation of the test stimulus in the retardation-ofacquisition test (Experiment 2). Tests followed feature-negative training in which $\mathrm{X}$ and $\mathrm{A}$ were presented serially or simultaneously for $0,4,20$, or 100 pairings. Error bars represent $S E M$ s. 
owing to X's superior temporal contiguity with A. Such an outcome would have been consistent with Kehoe et al.'s (1981) report that temporal contiguity affected the maximum magnitude of second-order conditioning in a trace second-order procedure. Kehoe et al.'s conclusion may well be correct under some conditions, but the parameters of the present study may not have been sensitive to this distinction.

The conclusion that trial number and the degree of $\mathrm{X}-\mathrm{A}$ contiguity interact to determine behavioral control in the feature-negative paradigm must be tempered by consideration of at least one potential confound in the present experiments: Trial spacing was confounded with trial number. Given the large number of groups necessary for these experiments, we chose to ignore the potential role of this variable. Instead, we allowed trial spacing to decrease with increasing trial number. However, Maisiak and Frey (1977) observed conditioned inhibition to be reduced by massed trial spacing. In contrast, the trial massing that was a byproduct of the many condition in the present research was accompanied by increased conditioned inhibition. Hence, the potential effects of this confound would have worked against the pattern of results observed in these experiments.

In summary, as $\mathrm{X}-\mathrm{A}$ trial number and temporal contiguity increased, less second-order conditioning and more conditioned inhibition was observed. Intuitively, this is not surprising, because it is reasonable that A must first come to predict the outcome before its associate, X, can come to be associated with the absence (or decrease in likelihood) of the expected outcome. However, as was stated, there are few formal models that are able to account for the present transition from second-order conditioning to conditioned inhibition in a principled fashion. The exceptions that explicitly address this topic are those of McLaren and Mackintosh (2000), Sutton and Barto (1981), and Wagner (1981). In each of these models, through different proximate mechanisms, $\mathrm{X}$ becomes associated with $\mathrm{A}$, which in turn becomes associated with the US. When presented early in training, $\mathrm{X}$ indirectly retrieves the US representation, leading to second-order excitatory conditioned responding. (This property of Wagner's model has received little attention, but see Mazur \& Wagner, 1982, for a discussion.) However, with more X-A trials, $\mathrm{X}$ develops an inhibitory association with the US by being paired with its omission in the presence of its expectation based on A. It seems that these models anticipate that the transition from second-order conditioning to conditioned inhibition should be faster for simultaneous than for serial $\mathrm{X}-\mathrm{A}$ pairings, because simultaneous pairings should enhance the rate of learning of the excitatory $\mathrm{X}-\mathrm{A}$ and inhibitory X-US associations.

The second-order conditioning observed prior to the development of conditioned inhibition is representative of a general challenge to learning investigators. In the last 30 years or so, a class of conditioning protocols that has had disproportionate impact on thinking within the field of learning has involved competition between cues. In cue competition preparations, the response strength to a target cue $(\mathrm{X})$ varies inversely with reinforcement of its associate (A). Examples of this inverse correlation between reinforcement of $\mathrm{A}$ and response potential of $\mathrm{X}$ include overshadowing (Pavlov, 1927, pp. 142-143, 269-270), blocking (Kamin, 1969), relative validity treatments (Wagner, Logan, Haberlandt, \& Price, 1968), and the recovery from these phenomena produced by posttraining extinction of A (Blaisdell, Gunther, \& Miller, 1999; Cole, Barnet, \& Miller, 1995; Kaufman \& Bolles, 1981). Conditioned inhibition and its extinction through nonreinforced presentations of A (e.g., Miller \& Schachtman, 1985) could be seen as additional examples of cue competition if response potential is defined broadly to include negative values representing inhibitory behavior (Rescorla \& Wagner, 1972; Wagner \& Rescorla, 1972).

Despite their impact on our most fundamental assumptions about the nature of associative learning, most of the aforementioned cue competition preparations have also been observed to yield the opposite behavioral result. That is, responding to a target cue can vary directly with reinforcement of its associate. Examples include potentiation (operationally identical to overshadowing; Rusiniak, Hankins, Garcia, \& Brett, 1979), augmentation (operationally identical to blocking; Batson \& Batsell, 2000), and second-order conditioning (operationally identical to conditioned inhibition). Moreover, posttraining extinction of the associate (A) can reduce response potential to $\mathrm{X}$ following potentiation, augmentation, and second-order conditioning treatments (e.g., Durlach \& Rescorla, 1980). Systematic attempts to identify the variables determining whether a given training protocol will result in cue competition, as opposed to cue facilitation, have been sporadic. Using the compound paradigm perhaps best known for yielding opposite behavioral outcomes, the featurenegative protocol, in the present experiments we investigated just two of the many possible interacting factorsnumber of compound trials and the temporal relationship of elements. Much work remains to be done in this area.

\section{REFERENCES}

BARnet, R. C., \& Miller, R. R. (1996). Temporal encoding as a determinant of inhibitory control. Learning \& Motivation, 27, 73-91.

BATSON, J. D., \& BATSELl, W. R., JR. (2000). Augmentation, not blocking, in an $\mathrm{A}+/ \mathrm{AX}+$ flavor-conditioning procedure. Psychonomic Bulletin \& Review, 7, 466-471.

BLAISDELl, A. P., GunTHER, L. M., \& Miller, R. R. (1999). Recovery from blocking achieved by extinguishing the block stimulus. Animal Learning \& Behavior, 27, 63-76.

Cole, R. P., BARnet, R. C., \& Miller, R. R. (1995). Effect of relative stimulus validity: Learning or performance deficit? Journal of Experimental Psychology: Animal Behavior Processes, 21, 293-303.

Durlach, P. J., \& Rescorla, R. A. (1980). Potentiation rather than overshadowing in flavor-aversion learning: An analysis in terms of within-compound associations. Journal of Experimental Psychology: Animal Behavior Processes, 6, 175-187.

Gallistel, C. R., \& GibBON, J. (2000). Time, rate, and conditioning. Psychological Review, 107, 289-344.

GiBBON, J., \& BALSAM, P. (1981). Spreading association in time. In C. M. Locurto, H. S. Terrace, \& J. Gibbon (Eds.), Autoshaping and conditioning theory (pp. 219-253). New York: Academic Press.

Gibbs, C. M., Cool, V., Land, T., Kehoe, E. J., \& Gormezano, I. 
(1991). Second-order conditioning of the rabbit's nictitating membrane response: Interstimulus interval and frequency of CS-CS pairings. Integrative Physiological \& Behavioral Science, 26, 282-295.

HerendeEN, D., \& ANDERSON, D. C. (1968). Dual effects of a secondorder conditioned stimulus: Excitation and inhibition. Psychonomic Science, 13, 15-16.

Holland, P. C. (1985). The nature of conditioned inhibition in serial and simultaneous feature negative discriminations. In R. R. Miller \& N. E. Spear (Eds.), Information processing in animals: Conditioned inhibition (pp. 267-297). Hillsdale, NJ: Erlbaum.

Holland, P. C., \& LamarRe, J. (1984). Transfer of inhibition after serial and simultaneous discrimination training. Learning \& Motivation, 15, 219-243.

Holland, P. C., \& Rescorla, R. A. (1975). Second-order conditioning with food as the unconditioned stimulus. Journal of Comparative \& Physiological Psychology, 88, 459-467.

KAMIN, L. J. (1969). Predictability, surprise, attention, and conditioning. In B. A. Campbell \& M. R. Church (Eds.), Punishment and aversive behavior (pp. 279-296). New York: Appleton-Century-Crofts.

Kaufman, M. A., \& Bolles, R. C. (1981). A nonassociative aspect of overshadowing. Bulletin of the Psychonomic Society, 18, 318-320.

Kehoe, E. J., Feyer, A.-M., \& Moses, J. L. (1981). Second-order conditioning of the rabbit's nictitating membrane response as a function of the CS2-CS1 intervals and CS1-US. Animal Learning \& Behavior, 9, 304-315.

LAMARRE, J., \& Holland, P. C. (1987). Transfer of inhibition after serial feature negative discrimination training. Learning \& Motivation, 18, 319-342.

LIEBERMAN, D. A. (2000). Learning: Behavior and cognition. Belmont, CA: Wadsworth.

Mackintosh, N. J. (1975). A theory of attention: Variations in the associability of stimuli with reinforcement. Psychological Review, 82, 276-298.

MAISIAK, R., \& FREY, P. W. (1977). Second-order conditioning: The importance of stimulus overlap on second-order trials. Animal Learning \& Behavior, 5, 309-314.

Mazur, J. E., \& Wagner, A. R. (1982). An episodic model of associative learning. In M. L. Commons, R. J. Herrnstein, \& A. R. Wagner (Eds.), Quantitative analyses of behavior: Vol. 3. Acquisition (pp. 339). Cambridge, MA: Ballinger.

McLaren, I. P. L., \& Mackintosh, N. J. (2000). An elemental model of associative learning: I. Latent inhibition and perceptual learning. Animal Learning \& Behavior, 28, 211-246.

Miller, R. R., \& Matzel, L. D. (1988). The comparator hypothesis: A response rule for the expression of associations. In G. H. Bower (Ed.), The psychology of learning and motivation (Vol. 22, pp. 51-92). San Diego: Academic Press.

Miller, R. R., \& Schachtman, T. R. (1985). Conditioning context as an associative baseline: Implications for response generation and the nature of conditioned inhibition. In R. R. Miller \& N. E. Spear (Eds.), Information processing in animals: Conditioned inhibition (pp. 5188). Hillsdale, NJ: Erlbaum.

PAPINI, M. R. (2002). Comparative psychology: Evolution and development of behavior. Englewood Cliffs, NJ: Prentice-Hall.

Papini, M. R., \& BitTerman, M. E. (1993). The two-test strategy in the study of inhibitory conditioning. Journal of Experimental Psychology: Animal Behavior Processes, 19, 342-352.

Pavlov, I. P. (1927). Conditioned reflexes (G. V. Anrep, Trans.). London: Oxford University Press.

Pearce, J. M. (1987). A model for stimulus generalization in Pavlovian conditioning. Psychological Review, 94, 61-73.

Pearce, J. M., \& HALl, G. (1980). A model for Pavlovian conditioning: Variations in the effectiveness of conditioned but not unconditioned stimuli. Psychological Review, 87, 532-552.

Rashotte, M. E., Marshall, B. S., \& O’Connell, J. M. (1981). Sig- naling functions of the second-order CS: Partial reinforcement during second-order conditioning of the pigeon's keypeck. Animal Learning \& Behavior, 9, 253-260.

ResCorla, R. A. (1969). Pavlovian conditioned inhibition. Psychological Bulletin, 72, 77-94.

RESCORLA, R. A. (1973). Second-order conditioning: Implications for theories of learning. In F. J. McGuigan \& D. B. Lumsden (Eds.), Contemporary approaches to conditioning and learning (pp. 127-150). Washington, DC: Winston.

RESCORLA, R. A. (1982). Simultaneous second-order conditioning produces S-S learning in conditioned suppression. Journal of Experimental Psychology: Animal Behavior Processes, 8, 23-32.

RESCORLA, R. A. (1985). Conditioned inhibition and facilitation. In R. R. Miller \& N. E. Spear (Eds.), Information processing in animals: Conditioned inhibition (pp. 299-326). Hillsdale, NJ: Erlbaum.

Rescorla, R. A., \& Wagner, A. R. (1972). A theory of Pavlovian conditioning: Variations in the effectiveness of reinforcement and nonreinforcement. In A. H. Black \& W. F. Prokasy (Eds. ), Classical conditioning II: Current research and theory (pp. 64-99). New York: Appleton-Century-Crofts.

Rizley, R. C., \& Rescorla, R. A. (1972). Associations in second-order conditioning and sensory preconditioning. Journal of Comparative \& Physiological Psychology, 81, 1-11.

RusiniaK, K. W., Hankins, W. G., Garcia, J., \& Brett, L. P. (1979) Flavor-illness aversions: Potentiation of odor by taste in rats. Behavioral \& Neural Biology, 25, 1-17.

SPENCE, K. W. (1936). The nature of discrimination learning in animals. Psychological Review, 43, 427-449.

SutTon, R. S., \& BARTO, A. G. (1981). Toward a modern theory of adaptive networks: Expectation and prediction. Psychological Review, 88, 135-171.

WAGNER, A. R. (1981). SOP: A model of automatic memory processing in animal behavior. In N. E. Spear \& R. R. Miller (Eds.), Information processing in animals: Memory mechanisms (pp. 5-47). Hillsdale, NJ: Erlbaum.

Wagner, A. R., Logan, F. A., Haberlandt, K., \& Price, T. (1968). Stimulus selection and a modified continuity theory. Journal of Experimental Psychology, 76, 171-180.

WAGNER, A. R., \& RESCORLA, R. A. (1972). Inhibition in Pavlovian conditioning: Application of a theory. In R. A. Boakes \& M. S. Halliday (Eds.), Inhibition and learning (pp. 301-336). New York: Academic Press.

Yin, H., Barnet, R. C., \& Miller, R. R. (1994). Second-order conditioning and Pavlovian conditioned inhibition: Operational similarities and differences. Journal of Experimental Psychology: Animal Behavior Processes, 20, 419-442.

\section{NOTES}

1. Holland (1985), however, has argued that the type of inhibition that results from the serial procedure is fundamentally different from that which results from the simultaneous procedure. Calling the former negative occasion setting, he has shown empirical dissociations in the effects of posttraining treatments of $\mathrm{A}$ and $\mathrm{X}$ that result from the simultaneous/serial distinction. By operationally defining conditioned inhibition as a stimulus that passes both tests, we here are ignoring the question of whether there are fundamentally distinct subclasses of conditioned inhibitors.

2. We are referring to the stimuli as $X$ and $A$, instead of clicks and light, respectively, to facilitate ease of discussion with respect to the introduction and the General Discussion section.

(Manuscript received October 24, 2003; revision accepted for publication December 22, 2003.) 